\title{
Laparoscopic local extraperitoneal para-aortic lymphadenectomy: Description of a novel technique
}

\author{
XUYIN ZHANG, JINGXIN DING and KEQIN HUA \\ Department of Obstetrics and Gynecology, Obstetrics and Gynecology Hospital, \\ Fudan University, Shanghai 200090, P.R. China
}

Received June 2, 2016; Accepted November 29, 2016

DOI: $10.3892 / \mathrm{mco} .2017 .1136$

\begin{abstract}
The aim of the present study was to introduce and describe a novel technique, which has been termed 'laparoscopic local extraperitoneal para-aortic lymphadenectomy', and to evaluate its feasibility and safety. In this retrospective case study, a series of 21 patients were selected who underwent laparoscopic local extraperitoneal para-aortic lymphadenectomy for gynecological malignancies between March and August 2014 at the Obstetrics and Gynecology Hospital of Fudan University, Shanghai, China. All the surgical procedures were performed by the same operational team. Patients' data were collected retrospectively, and the characteristics included patient age, body mass index (BMI), tumor stage, tumor grade, blood loss, operative time (laparoscopic local extraperitoneal para-aortic lymphadenectomy), length of hospital stay, intraoperative and postoperative complications, pathology, lymph node count and lymph node status. A total of 21 patients underwent laparoscopic local extraperitoneal para-aortic lymphadenectomy. The median patient age was 52 years (range, 45-71 years); the median BMI was $24.8 \mathrm{~kg} / \mathrm{m}^{2}$ (range, $22.2-32.4 \mathrm{~kg} / \mathrm{m}^{2}$ ); the median operating time for the para-aortic lymphadenectomy was 70 min (range, 58-95 min); and the median estimated blood loss of the total surgery was $200 \mathrm{ml}$ (range, 100-600 ml). No patient required a blood transfusion during the operation. The median length of hospital stay was 7 days (range, 5-9 days), and the median number of para-aortic lymph nodes was measured as 12 (range, 7-17). Postoperative complications included one patient with chylous ascites, who responded to conservative management with intravenous somatostatin. In conclusion, the present study has demonstrated that laparoscopic local extraperitoneal para-aortic lymphadenectomy, which avoids interference of the bowels during surgery, is safe and practical.
\end{abstract}

Correspondence to: Professor Jingxin Ding or Professor Keqin Hua, Department of Obstetrics and Gynecology, Obstetrics and Gynecology Hospital, Fudan University, 419 Fang Xie Road, Shanghai 200090, P.R. China

E-mail: djxdd@sina.com

E-mail: huakeqinjiaoshou@163.com

Key words: laparoscopic para-aortic lymphadenectomy, extraperitoneal surgery, endometrial cancer, cervical cancer

\section{Introduction}

The advancement of surgical instruments and equipment over the last 5 years has expanded and confirmed the advantages of minimally invasive surgery in the treatment of numerous gynecological diseases, particularly in cases of gynecological malignancies. Numerous studies have confirmed that laparoscopic techniques associated with various gynecological oncological conditions yield improved results compared with surgical and oncological outcomes of the abdominal staging, with the advantages of shorter hospital stays, fewer postoperative adhesions and an improved quality of life (1-3).

Since laparoscopic extraperitoneal para-aortic lymphadenectomy was described by Vasilev and McGonigle in 1996 (1), it has been accepted in the literature that laparoscopic extraperitoneal para-aortic lymphadenectomy may be performed in patients. This approach theoretically combines the benefits of laparoscopy with the extraperitoneal approach. It leads to a decrease in the risk of direct bowel injury, adhesion formation, and wound complications, and possibly decreases the length of hospital stay and treatment delay (2).

However, in the majority of cases of laparoscopic para-aortic lymphadenectomy, total laparoscopic pelvic lymphadenectomy and hysterectomy with bilateral salpingo-oophoerctomy (TLH\&BSO) will be performed on the patients, and occasionally even radical hysterectomy, which are all transperitoneal operations. Furthermore, the conventional incision positions are neither suitable nor easy for surgeons to reach.

In the present study, a novel technique has been introduced and described, which is termed 'laparoscopic local extraperitoneal para-aortic lymphadenectomy', and its feasibility and safety were evaluated.

\section{Materials and methods}

The present retrospective case study included a series of 21 patients, who underwent laparoscopic local extraperitoneal para-aortic lymphadenectomy for gynecological malignancies between March and August 2014 in the Obstetrics and Gynecology Hospital of Fudan University, Shanghai, China.

Diagnoses for the patients included ovarian cancer, endometrial carcinoma with high-risk factors (i.e., myometrial invasion $>50 \%$, large tumor diameter, and grade 3 or non-endometrioid pathology) and cervical cancer with 
common iliac nodal involvement. All the surgical procedures were performed by the same operational team. Patients' data were collected retrospectively, and the characteristics included patient age, body mass index (BMI), tumor stage and grade, blood loss, operative time (laparoscopic local extraperitoneal para-aortic lymphadenectomy), length of hospital stay, intraoperative and postoperative complications, pathology, lymph node count and lymph node status.

Once the patient had been anesthetized, she was placed in the Trendelenburg and lithotomy position. The operator was positioned to the left of the patient, while the first assistant stood on the right of the patient. A $10-\mathrm{mm}$ trocar was sub-umbilically inserted for the introduction of the camera and intraperitoneal inspection. A carbon dioxide pneumoperitoneum was subsequently generated, keeping the intra-abdominal pressure $<14 \mathrm{mmHg}$. A 30-degree laparoscope was the introduced, and the peritoneal cavity was inspected. Associated with the inspection, ancillary trocars were placed within the patient in the supine position, as follows: i) A $10-\mathrm{mm}$ trocar at the McBurney's point; ii) a 5-mm trocar at the right lateral of the umbilicus, $4 \mathrm{~cm}$ in distance; and iii) two further $5-\mathrm{mm}$ trocars at the left side of the patient, opposite to the right two trocars (Fig. 1). These procedures conformed with those of TLH\&BSO, also even including radical hysterectomy, starting with conventional transperitoneal laparoscopy, as has been widely reported.

Subsequently, an additional 10-mm trocar was placed $4 \mathrm{~cm}$ above the pubic symphysis, and the laparoscope was inserted in this suprapubic port (Fig. 2). The operator and first assistant changed their positions, turning around and facing the TV monitor, which was moved to the head of the patient. The present authors consider that this position makes the following operation easier compared with the conventional position.

The local peritoneum on top of the lower aorta was incised using a Harmonic scalpel (Ethicon Endosurgery, Inc.; Johnson \& Johnson, Cincinatti, OH, USA), the peritoneum was raised with atraumatic graspers, and the laparoscope was then inserted beneath the peritoneum. Subsequently, the exposure of aorta was optimized up to the left renal vein (Fig. 3). In this procedure, the duodenum and the small intestine in the peritoneal cavity were kept isolated from the operation area by this local extraperitoneal approach. The nodal tissue was subsequently gently dissected.

With the identification of the inferior mesenteric artery and the left ureter, the left para-aortic lymphadenectomy included inframesenteric lymphadenectomy (the aorta up to the level of the inferior mesenteric artery) and infrarenal lymphadenectomy (the aorta up to the level of the left renal vein). The right para-aortic lymphadenectomy included the lymph nodes around the vena cava up to the right ovarian vein.

Since the greater omentum is located at the upper abdomen, the improved position also facilitated the operation in cases with omentectomy.

\section{Results}

A total of 21 patients underwent laparoscopic local extraperitoneal para-aortic lymphadenectomy, including 14 with endometrioid endometrial cancer, two with cervical cancer and five with ovarian cancer. The median patient age was

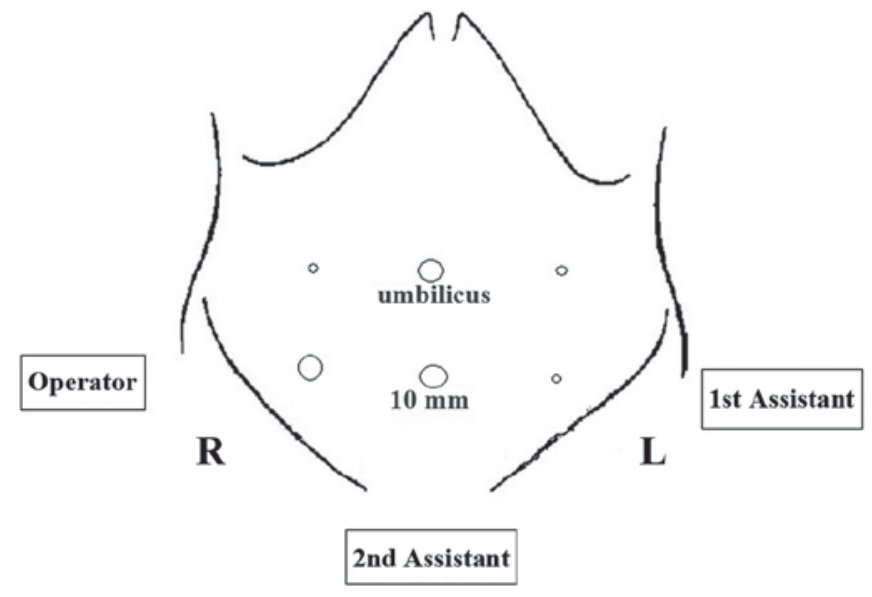

Figure 1. The position of trocar incision of conventional transperitoneal laparoscopic pelvic lymphadenectomy and hysterectomy, also even including radical hysterectomy. $\mathrm{L}$, left; $\mathrm{R}$, right.

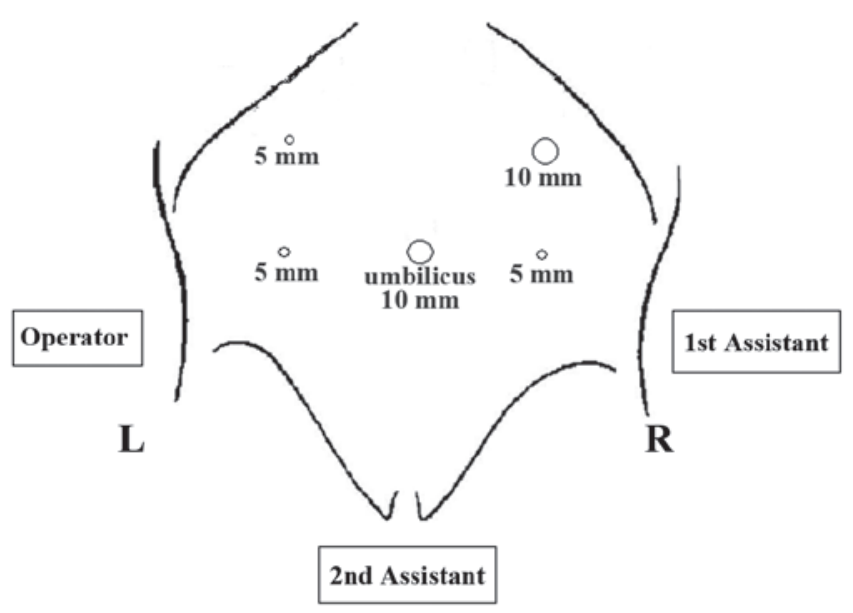

Figure 2. The position of laparoscopic local extraperitoneal para-aortic lymphadenectomy. $\mathrm{L}$, left; $\mathrm{R}$, right.

52 years (range, $45-71$ years). The median body mass index was $24.8 \mathrm{~kg} / \mathrm{m}^{2}$ (range, 22.2-32.4 kg/m²) (Table I). All patients tolerated the procedure and positioning well.

All patients with endometrial cancer were surgically treated using TLH\&BSO. The patients with cervical cancer were managed by radical hysterectomy, with or without BSO. The three cases of ovarian cancer underwent cytoreductive surgery. All patients underwent pelvic lymphadenectomy. The median operating time of para-aortic lymphadenectomy was $70 \mathrm{~min}$ (range, 58-95 min). The median estimated blood loss of the total surgery was $200 \mathrm{ml}$ (range, 100-600 ml). No patient required a blood transfusion during the operation. The median length of hospital stay was 7 days (range, 5-9 days). There were no conversions or intraoperative complications in any of the patients. The median number of para-aortic lymph nodes was 12 (range, 7-17), and the mean number of pelvic lymph nodes was 22 (range, 20-25). Positive aortic nodes metastasis was detected in one patient with ovarian cancer.

Postoperative complications included one patient with chylous ascites, who responded to conservative management with intravenous somatostatin. 


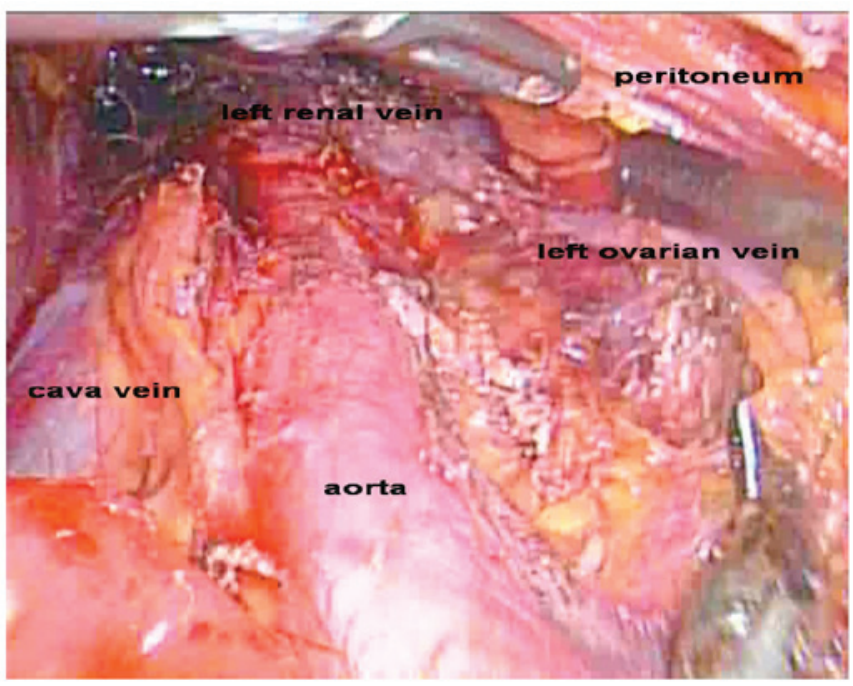

Figure 3. The local peritoneum was incised and raised with atraumatic graspers. The exposure of the left renal vein is illustrated.

\section{Discussion}

The present study demonstrated the effectiveness and the safety of laparoscopic local extraperitoneal para-aortic lymphadenectomy in patients with gynecological cancer.

There are two advantages associated with this novel procedure compared with the methods previously reported. The first advantage was the positional change of the laparoscope to the suprapubic port, and the operator and first assistant turning around to face the TV located at the head of the patient, which facilitates the performance of this operation compared with the conventional position. The para-aortic lymph nodes were located at the upper abdomen, and laparoscopic inframesenteric lymphadenectomy (the aorta up to the level of inferior mesenteric artery) was commonly performed with the conventional position and umbilicus trocar for the laparoscope. However, infrarenal lymphadenectomy (the aorta up to the level of left renal vein) was more difficult, as the lymph nodes are located immediately under the umbilicus, and the operation of the laparoscopic clamp was aligned vertically to the abdominal wall. It is well established that, the smaller the angle between the laparoscopic clamp and abdominal wall, the more difficult will be the operation. Since the greater omentum is located at the upper abdomen, the improved position also facilitated the operation in cases involving an omentectomy.

The second advantage was that the novel procedure reported in the present study is different from total extraperitoneal para-aortic lymphadenectomy. Since para-aortic lymphadenectomy usually follows the transperitoneal pelvic lymphadenectomy, total extraperitoneal para-aortic lymphadenectomy does not appear to have specific superiority, and the extraperitoneal laparoscopic approach should be considered for endometrial cancer staging in patients with a BMI $\geq 35 \mathrm{~kg} / \mathrm{m}^{2}$ (3). Laparoscopic local extraperitoneal para-aortic lymphadenectomy, particularly infrarenal lymphadenectomy, may facilitate the operation and avoid injury to the duodenum.

The median number of harvested para-aortic lymph nodes in the present study was 12 (range, 7-17). The lymph node numbers obtained in this study are similar to those in
Table I. Patient characteristics.

\begin{tabular}{lc}
\hline Characteristic (total no. of patients, $\mathrm{n}=21)$ & $\mathrm{n}$ \\
\hline Endometrial cancer & 14 \\
FIGO stage & 10 \\
Ib & 4 \\
IIa & \\
Grade & 11 \\
2 & 3 \\
3 & 2 \\
Cervical cancer & \\
FIGO stage & 1 \\
Ib2 & 1 \\
IIa1 & 5 \\
Ovarian cancer & \\
FIGO stage & 1 \\
IIb & 2 \\
IIIa & 2 \\
IIIb & $52(45-71)$ \\
Age (years), median (range) & $24.8(22.2-32.4)$ \\
BMI (kg/m ${ }^{2}$ ), median (range) & \\
\end{tabular}

BMI, body mass index; FIGO, international federation of gynecology and obstetrics.

previously published reports. For example, Escobar et al (4) reported para-aortic lymph node sampling lymphadenectomy performed through a single $2-3 \mathrm{~cm}$ umbilical incision using a single-port device, and the lymph nodes count was 6 (range, 2-14). A similar median number of para-aortic nodes (14; range, 12-24) was revealed by Kavallaris et al (5) by means of a standardized technique of laparoscopic para-aortic lymphadenectomy in gynecological cancer.

A previous study demonstrated that single-port laparoscopic para-aortic lymphadenectomy was associated with only a relatively postoperative hidden umbilical scar, and resulted in shorter hospital stays, an improved quality of life and surgical and oncological outcomes that were comparable with those of abdominal staging (6). However, the major disadvantage of single-port surgery is the collision of instruments, and the requirement for specialized instruments, such as the single-port device.

The present study did have a number of limitations, due to its retrospective nature, the small number of patients involved, and lack of randomization. In conclusion, this case report has demonstrated the feasibility of performing a laparoscopic local extraperitoneal para-aortic lymphadenectomy in gynecological cancers. Studies involving a bigger sample size are necessary, and are eagerly awaited, to help to determine the long-term risks and benefits.

\section{Acknowledgements}

The present study was supported by the Science and Technology Committee of Shanghai (grant no. 20144Y0096). 


\section{References}

1. Vasilev SA and McGonigle KF: Extraperitoneal laparoscopic para-aortic lymph node dissection. Gynecol Oncol 61: 315-320, 1996.

2. Iacoponi S, De Santiago J, Diestro MD, Hernandez A and Zapardiel I: Single-port laparoscopic extraperitoneal para-aortic lymphadenectomy. Int J Gynecol Cancer 23: 1712-1716, 2013.

3. Pakish J, Soliman PT, Frumovitz M, Westin SN, Schmeler KM, Reis RD, Munsell MF and Ramirez PT: A comparison of extraperitoneal versus transperitoneal laparoscopic or robotic para-aortic lymphadenectomy for staging ofendometrial carcinoma. Gynecol Oncol 132: 366-371, 2014.

4. Escobar PF, Fader AN, Rasool N and Espalliat LR: Single-port laparoscopic pelvic and para-aortic lymph node sampling or lymphadenectomy: Development of a technique and instrumentation. Int J Gynecol Cancer 20: 1268-1273, 2010.

5. Kavallaris A, Kalogiannidis I, Chalvatzas N, Hornemann A, Bohlmann MK and Diedrich K: Standardized technique of laparoscopic pelvic and para-aortic lymphadenectomy in gynecologic cancer optimizes the perioperative outcomes. Arch Gynecol Obstet 283: 1373-1380, 2011.

6. Gouy S, Uzan C, Scherier S, Gauthier T, Bentivegna E, Kane A, Morice P and Marchal F: Single-port laparoscopy and extraperitoneal para-aortic lymphadenectomy for locally advanced cervical cancer: Assessment after 52 consecutive patients. Surg Endosc 28: 249-256, 2014. 